\title{
The Wiring Economy Principle for Designing Inference Networks
}

\author{
Lav R. Varshney, Member, IEEE
}

\begin{abstract}
The wiring economy principle in neuroscience has explained many experimentally observed properties of neuronal networks by asserting the need to keep the axons and dendrites that connect neurons small in length. Just like neuronal networks, many distributed systems are physical constructs that incur deployment and maintenance costs for their communication infrastructure. Taking wiring economy as a design goal for engineering systems that perform distributed coordination and inference, this paper formulates and studies the tradeoff between performance and wiring cost. It is shown that separated communication topology design and physical node placement yields optimal design. Designing optimal networks is shown to be NP-complete. The natural relaxation to the integer network design problem is shown to be a reverse convex program. Small optimal networks are computed. Optimally placed random network topologies are demonstrated to have good performance.
\end{abstract}

Index Terms -Distributed inference, network design, wiring.

\section{INTRODUCTION}

$\mathbf{T}$ HE WIRING economy principle in neuroscience states that wiring minimization plays an important role in determining brain organization [1]. Dating back to the work of Cajal in the nineteenth century [2], this hypothesis has allowed theoretical explanation and prediction of several experimentally observed features of brain organization such as topographic maps [3], [4], dendritic and axonal arbor sizes [5], [6], and spatial layout of neurons and their connectivity patterns [7]-[12]. Whether primarily due to wiring volume, metabolic cost, signal delay and attenuation, or developmental overhead, it is clear that wiring cost increases with the distance between connected neurons.

Neuronal networks do not just incur cost but also provide significant information processing functionality. Nervous systems perform many control and computation tasks: one notable example is sensory signal processing to infer whether the concentration of noxious chemicals is dangerous and then coordinate motor control in response [13] (as in the

Manuscript received August 13, 2012; revised January 17, 2013. This material is based upon work supported by the National Science Foundation under a Graduate Research Fellowship, and Grants 0729069, 0836720, and 0801549, and a Siemens Corporate Research Grant, "Advanced Control Methods for Complex Networked Systems." This work is based in part on a thesis submitted in partial fulfillment of the requirements for the degree of Doctor of Philosophy in the Department of Electrical Engineering and Computer Science at the Massachusetts Institute of Technology and was presented in part at the American Control Conference, Baltimore, MD, July 2010 .

L. R. Varshney was with the Department of Electrical Engineering and Computer Science, the Laboratory for Information and Decision Systems, and the Research Laboratory of Electronics, Massachusetts Institute of Technology. He is now with the IBM Thomas J. Watson Research Center (e-mail: varshney@alum.mit.edu).

Digital Object Identifier 10.1109/JSAC.2013.130611. neuronal network of the nematode C. elegans [14]). Neuronal networks balance wiring cost against information processing functionality [1], [15], [16]; indeed some argue that desired functionality directly determines physical anatomy through the wiring economy principle [17].

Engineered network systems also take physical form, incurring costs of deploying and maintaining the physical infrastructure of communication network links. Whereas most previous works that consider network systems have ignored their spatial aspects, cf. [18], [19], this paper considers tradeoffs between functionality and spatially-driven wiring costs. Taking insight from neuronal networks, which have faced evolutionary pressure to have efficient physical organization, this paper develops techniques to design communication network structures that have good functionality but that economize wiring.

As typical examples of systems that can be engineered using the wiring economy principle, consider the following:

- a wireline sensor network that must infer whether the outside temperature is appropriate for irrigation [20], built from sensor nodes and wires to connect them;

- an integrated circuit for decoding a repetition code to infer whether the transmitted message is zero or one, built from logic gates and wires to connect them; and

- a fault-tolerant grid computing infrastructure that must synchronously distribute work and recombine redundant computations, built from individual processors and optical fiber links to connect them.

The information processing functionality required in all of these systems is consensus and distributed inference. Consequently we study algorithms for these two problems and use speeds of convergence as measures of functionality. Such notions of functionality have previously been developed in the network science literature under the broad heading of synchronizability [21].

Main results are as follows. First we prove a separation principle such that optimal networks are designed by solving two subproblems: designing the graph connectivity on the basis of functionality considerations and then placing the graph in physical space on the basis of the wiring economy principle, thereby recovering the neuroscientific principle of [17] in a precise mathematical setting. Next we prove a new computational complexity result for network design that shows optimal design is NP-complete. Then we develop a natural reverse convex relaxation for the optimal design problem for computationally efficient design. We use random matrix theory to demonstrate that random graph topologies are close to optimal. Finally we exactly compute many small optimal networks and give insight into the general nature of solutions. 


\section{DEFINING WiRING COSTS AND FunCtionality}

Drawing on the physical properties of wires and the nature of iterative inference algorithms, let us define the precise mathematical form of wiring cost and convergence speed. Before proceeding, some algebraic graph theory is reviewed.

\section{A. Algebraic Graph Theory}

Eigenvalues and eigenvectors of certain matrices associated with graphs are of central importance in understanding properties of dynamical systems defined on those graphs.

Let $G=(V, E)$ be a graph with vertex set $V$ of cardinality $n$ and edge set $E \subseteq V \times V$. Let $A$ be the adjacency matrix of the graph. Let $d_{j}$ indicate the degree of vertex $j$ and let $D$ be the degree matrix of the graph, which takes value $d_{j}$ along the diagonal and value 0 otherwise. The Laplacian matrix of a graph, $L$, satisfies $L=D-A$. The eigenvalues of $L$ are denoted $\lambda_{1}(L) \leq \lambda_{2}(L) \leq \cdots \leq \lambda_{n}(L)$. Since $L$ is symmetric, all of its eigenvalues are real and eigenvectors corresponding to different eigenvalues are orthogonal.

The Laplacian matrix of every graph is positive semidefinite and its eigenvalues are non-negative. Further, since the row sums of $L$ are all zero, the all-ones vector $\overrightarrow{1}$ is an eigenvector with eigenvalue $\lambda_{1}=0$. The multiplicity of the eigenvalue 0 determines the number of connected components in the graph; if $\lambda_{2}>0$, then the graph is a single connected component.

The Courant-Fischer min-max theorems endow the eigenvalues with certain optimality properties. In particular,

$$
\lambda_{2}=\min _{\left\{\vec{x} \in \mathbb{R}^{n}: \vec{x} \neq \overrightarrow{0} \text { and } \vec{x} \perp \overrightarrow{1}\right\}} \frac{\langle\vec{x}, L \vec{x}\rangle}{\langle\vec{x}, \vec{x}\rangle},
$$

where $\overrightarrow{0}$ is the all-zero vector. This second smallest eigenvalue $\lambda_{2}$ is called the algebraic connectivity of the graph. The largest eigenvalue satisfies

$$
\lambda_{n}=\max _{\left\{\vec{x} \in \mathbb{R}^{n}: \vec{x} \neq \overrightarrow{0} \text { and } \vec{x} \perp \overrightarrow{1}\right\}} \frac{\langle\vec{x}, L \vec{x}\rangle}{\langle\vec{x}, \vec{x}\rangle} .
$$

The largest eigenvalue $\lambda_{n}$ is called the spectral radius of the graph Laplacian. The eigenratio is defined as $\rho=\lambda_{2} / \lambda_{n}$.

\section{B. Wiring Costs}

Reconsider the example from the introduction: a communication network embedded in an integrated circuit. System performance depends on the electrical properties of the wiring such as resistance, conductance, and inductance [22]. Load for driving gates increases with wire capacitance; signal delay increases with wire resistance, capacitance, and inductance; and signal noise increases with inductive and capacitive coupling between wires. These electrical properties depend not only on wire material, but also on geometric properties such as length [23].

Wires are also directly costly [22], [24]-[27]. For example, Theis noted that "wiring has long been identified as an eventual limiter of integrated circuit performance, but the $\mathrm{Na}$ tional Technology Roadmap for Semiconductors (NTRS) now estimates that without radical material, design, or architectural innovations, this point will be reached [soon]" [28]. The cost of a wire in integrated circuits is quadratic in its length [29], similar to axon and dendrite cost in neuronal networks [11], [12]. Similarly for other kinds of wires in networks.

A physical network in Euclidean space is determined not only by logical connectivity but also by physical placement of nodes (and thereby physical lengths and locations of links). Hence a primary aspect of the problem formulation is embedding a logical graph structure in Euclidean space [30]. Following insight from neuroscience, an adjacency model for graph drawing - where the geometry respects the binary relations of adjacency/nonadjacency between vertices [31], [32] - is used. The wiring economy principle is implemented by drawing graphs in Euclidean space to minimize quadratic wiring cost.

Consider the wiring cost for a graph with adjacency matrix $A$, with elements $a_{i j} \in\{0,1\}$. Vertices are placed on the real line at $\vec{x}=\left(x_{1}, x_{2}, \ldots, x_{n}\right), x_{i} \in \mathbb{R}$. The total wiring cost, $W_{1}$ is then

$$
W_{1}=\frac{1}{2} \sum_{i=1}^{n} \sum_{j=1}^{n}\left(x_{i}-x_{j}\right)^{2} a_{i j} .
$$

If the network is to be drawn in two-dimensional Euclidean space with horizontal placement $\vec{x}$ and vertical placement $\vec{y}$, the wiring cost $W_{2}$ is

$$
W_{2}=\frac{1}{2} \sum_{i=1}^{n} \sum_{j=1}^{n}\left(x_{i}-x_{j}\right)^{2} a_{i j}+\left(y_{i}-y_{j}\right)^{2} a_{i j} .
$$

Likewise for three-dimensional cost $W_{3}$.

\section{Convergence Speed}

Consensus [33], [34] and inference [35], [36] are critical tasks for many engineered network systems, just as they are for nervous systems [13] and other natural networked systems [37], [38]. With these tasks, the faster the better. Hence we consider the convergence speeds of distributed consensus and distributed inference algorithms. Assuming signal propagation times are negligible, algorithmic convergence speeds are governed solely by the logical connectivity of nodes.

As a typical consensus algorithm, suppose each vertex $i$ in the graph has a state variable $\sigma_{i}(t) \in \mathbb{R}$ which is updated during each iteration $t$ of the algorithm. The initial state is

$$
\vec{\sigma}(t=0)=\left[\ell_{1}, \ldots, \ell_{n}\right]^{T} .
$$

Vertices iteratively pass messages to their neighbors in the network based on weighted averaging of the current state and keep what they pass as their state variable.

$$
\vec{\sigma}(t)=Z \vec{\sigma}(t-1)=Z^{t} \vec{\sigma}(0) \text { for } t \geq 1
$$

where $Z$ is the weight matrix of the algorithm and has the same sparsity pattern as the adjacency matrix $A$ of the network. The weight matrix is chosen so all edges have equal weight $\beta$ :

$$
Z=I-\beta L
$$

where $I$ is the identity matrix and $L$ is the graph Laplacian, with eigenvalues $\lambda_{1}(L) \leq \lambda_{2}(L) \leq \cdots \leq \lambda_{n}(L)$. 
If the network is connected and $\beta$ is bounded as $0<\beta<$ $2 / \lambda_{n}(L), \lim _{t \rightarrow \infty} \vec{\sigma}(t)$ exists and satisfies

$$
\lim _{t \rightarrow \infty} \sigma_{i}(t)=\frac{1}{n} \sum_{i=1}^{n} \sigma_{i}(0)=\frac{1}{n} \sum_{i=1}^{n} \ell_{i}=\bar{\ell},
$$

for all $i=1, \ldots, n$ [36]. The state variables $\sigma_{i}$ at every vertex surely converge to the same value.

The convergence speed of $\vec{\sigma}$ to $\bar{\ell}$ is characterized by the convergence factor

$$
r(Z)=\sup _{\vec{\sigma}(t) \neq \bar{\ell}} \frac{\|\vec{\sigma}(t+1)-\bar{\ell}\|_{2}}{\|\vec{\sigma}(t)-\bar{\ell}\|_{2}}
$$

and associated convergence time

$$
\tau(Z)=\frac{1}{\log (1 / r(Z))} .
$$

The goal will be to optimize $r(Z(\beta, L))$ through choice of parameter $\beta$ and network structure represented by $L$.

There is a broad class of iterative message-passing algorithms for consensus as above and for inference that proceed in a similar way [33]. In general, we would want to optimize their convergence factors.

\section{Separate Optimization of Connectivity AND PLACEMENT}

Having defined precise notions of wiring costs and of functionality as measured through consensus/inference convergence speed, now consider how to optimize them individually. Later in the section, we show there is no loss in optimality by separate optimization.

\section{A. Placement}

Placing nodes in Euclidean space to minimize quadratic wiring costs has an algebraic graph theory solution; this has previously been observed in neuroscience [11], [39].

Consider the wiring cost in the one-dimensional setting:

$$
\begin{aligned}
W_{1} & =\frac{1}{2} \sum_{i=1}^{n} \sum_{j=1}^{n}\left(x_{i}-x_{j}\right)^{2} a_{i j} \\
& =\sum_{i=1}^{n} x_{i}^{2} \sum_{j=1}^{n} a_{i j}-\sum_{j=1}^{n} \sum_{i: i \neq j} x_{i} x_{j} a_{i j} \\
& =\vec{x}^{T}(D-A) \vec{x}=\vec{x}^{T} L \vec{x} .
\end{aligned}
$$

Two non-triviality constraints should be imposed on the wiring cost minimizing placement. First, all nodes are required to not be coincident. Second, a normalization that $\vec{x}^{T} \vec{x}=1$ is imposed, so that nodes are not arbitrarily close to being coincident. This provides a measurement scale to the problem. Under these non-triviality constraints, the Courant-Fischer theorem implies that the placement $\vec{x}$ should be the unit eigenvector associated with $\lambda_{2}$ and that the incurred wiring cost is $\lambda_{2}$, for placement in one-dimensional Euclidean space.

If the network is to be drawn in two-dimensional Euclidean space with horizontal placement $\vec{x}$ and vertical placement $\vec{y}$, it follows from (4) that wiring cost is $W_{2}=\vec{x}^{T} L \vec{x}+\vec{y}^{T} L \vec{y}$. If a further non-triviality constraint that horizontal placement must be orthogonal to vertical placement is imposed, ${ }^{1}$ then the optimal placement is such that $\vec{x}$ is the eigenvector associated with $\lambda_{2}$ and $\vec{y}$ is the eigenvector associated with $\lambda_{3}$. The incurred cost is $\lambda_{2}+\lambda_{3}$. In three dimensions, the cost is $W_{3}=\vec{x}^{T} L \vec{x}+\vec{y}^{T} L \vec{y}+\vec{z}^{T} L \vec{z}$ and under optimal non-trivial placement, the cost is $W_{3}=\lambda_{2}+\lambda_{3}+\lambda_{4}$.

\section{B. Connectivity}

Finding the best logical graph structure for consensus or inference convergence speed requires optimizing the convergence factor, (3).

The optimal weight is

$$
\beta^{*}=\frac{2}{\lambda_{2}(L)+\lambda_{n}(L)},
$$

determined by $\lambda_{2}(L)$ and $\lambda_{n}(L)$ for any network structure $[36,(23)]$. The convergence factor with optimal weight $\beta^{*}$ is

$$
r(L)=\frac{1-\rho(L)}{1+\rho(L)}
$$

where $\rho(L)=\lambda_{2}(L) / \lambda_{n}(L)$ is the eigenratio [35, (6)]. Notice from (5) that minimizing $r(L)$ is equivalent to maximizing $\rho(L)$ and so the algorithm converges quickly on networks with large eigenratio.

On the other hand, the convergence speeds of several consensus algorithms related to the one presented are determined by the algebraic connectivity $\lambda_{2}(L)$; see e.g. [21], [33], [41], [42] and references therein. These algorithms converge quickly on networks with large algebraic connectivity.

Both algebraic connectivity $\lambda_{2}$ and eigenratio $\rho$ are notions of synchronizability [21]. A few previous works have considered optimizing $\lambda_{2}$ or $\rho$ through the choice of graph topology [35], [43]-[46]. Ramanujan graphs, a particular class of expander graphs, have extremal properties [35].

Kar et al. assert that the eigenratio is more sensitive to variations in the algebraic connectivity than variations in the spectral radius. Hence they develop topology constructions that optimize $\lambda_{2}$ [35]. Rad et al. demonstrate that graphs optimized for $\rho$ also have large $\lambda_{2}$ [21]. The sequel provides some treatment as to whether networks with large $\lambda_{2}$ also have large $\rho$, once wiring cost constraints are imposed.

\section{A Separation Principle}

To optimize the synchronizability of a communication network under the wiring economy principle, we want to bring placement and connectivity optimization together. In service of this goal, we show that there is a separation such that optimal networks are found by solving two subproblems: designing the graph connectivity and then placing the graph in physical space (for either notion of synchronizability).

Theorem 1: There is an optimal non-trivial placement of graph vertices in Euclidean space for any given choice of graph topology $A$ and so the topology design to determine

${ }^{1}$ If the network is to simultaneously detect, estimate, or control on a bandlimited field, a reason for imposing this non-triviality constraint may be related to sampling. Stable sampling requires the entire space to have reasonable sampling density [40]. 
$A$ and the placement of $A$ may be separated without loss of optimality.

Proof: Suppose the contrary that logical connectivity design $A$ influences the optimal placement algorithm. Then there would exist two different optimal placement algorithms $f_{1}$ and $f_{2}$ for some admissible matrices $A_{1}$ and $A_{2}$.

However by the Courant-Fischer theorem, for each admissible $A$ the same spectral placement algorithm $f$ is an optimal placement. Hence $f_{1}$ and $f_{2}$ are identical, and so the result follows by contradiction.

This theorem recovers the neuroscientific principle that desired functionality directly determines physical anatomy through the wiring economy principle [17], but in a precise and abstract setting applicable not only for understanding neuronal networks but also for designing engineered network systems.

\section{Functionality-Cost TradeofF}

We have established extremal network functionality in terms of either $\lambda_{2}$ or $\rho$, have established extremal wiring network cost in terms of $\left\{\lambda_{i}\right\}_{i=2}^{r+1}$, and have established a separationbased design principle. Now we consider the optimal tradeoff between functionality and cost.

\section{A. Formal Specification}

Due to Thm. 1, the network design problems reduce to the optimization of graph topology using algebraic graph theory.

Let $\Gamma$ be a graph with $n$ vertices and $L(\Gamma)$ its Laplacian. Also let $r, 1 \leq r<n$, be the dimension of the Euclidean space in which the network is to be built, e.g. $r=2$, or $r=3$. Then the set of graphs $\mathcal{G}$ that meet a wiring cost constraint $W_{r}$ under optimal placement is

$$
\mathcal{G}\left(W_{r}\right)=\left\{\Gamma: \sum_{i=2}^{r+1} \lambda_{i}(L(\Gamma)) \leq W_{r}\right\} .
$$

The design problems to be solved are as follows.

Problem 1: Optimize Algebraic ConNeCtivity with WIRING ECONOMY

Find the following functionality-cost function:

$$
s_{\lambda}\left(W_{r}\right)=\max _{G \in \mathcal{G}\left(W_{r}\right)} \lambda_{2}(L(G)) .
$$

Also find optimizing graphs

$$
G_{\lambda}^{*}\left(W_{r}\right)=\underset{G \in \mathcal{G}\left(W_{r}\right)}{\arg \max } \lambda_{2}(L(G)) .
$$

When $r=1$, the objective function and constraint coincide. Since they are directly opposed, there is no non-trivial tradeoff. Primary interest here, however, is in $r=2$ or $r=3$.

Problem 2: OptIMIZE EIgENRATIO WITH WIRING ECONOMY

Find the following functionality-cost function:

$$
s_{\rho}\left(W_{r}\right)=\max _{G \in \mathcal{G}\left(W_{r}\right)} \rho(L(G))=\max _{G \in \mathcal{G}\left(W_{r}\right)} \frac{\lambda_{2}(L(G))}{\lambda_{n}(L(G))} .
$$

Also find optimizing graphs

$$
G_{\rho}^{*}\left(W_{r}\right)=\underset{G \in \mathcal{G}\left(W_{r}\right)}{\arg \max } \rho(L(G))=\underset{G \in \mathcal{G}\left(W_{r}\right)}{\arg \max } \frac{\lambda_{2}(L(G))}{\lambda_{n}(L(G))} .
$$

Probs. 1 and 2 formalize optimization of convergence speed under the wiring economy principle.

\section{B. Some Related Tradeoffs}

There has been some previous work looking at tradeoffs between functionality and cost in physical networks. For spatial distribution networks, the relationship between the lengths of paths from each vertex to the root vertex and the sum of the lengths of all edges in the network is discussed in [19]. The tradeoff between algorithm performance and communication cost has also been discussed as a network design problem [41]. Ghosh and Boyd briefly discuss optimizing $\lambda_{2}$ when costly links may be added to a network [43].

\section{Deriving an Upper Bound}

The performance of a complete graph yields an upper bound on the tradeoff for the algebraic connectivity problem.

Theorem 2: Solutions to the algebraic connectivity problem, Prob. 1, satisfy

$$
s_{\lambda}\left(W_{r}\right) \leq \frac{W_{r}}{r},
$$

and there exists at least one network that achieves the bound with equality for any admissible $n$ and $r$.

Proof: Choose an admissible pair $n$ and $r$. The bound

$$
r \lambda_{2}(L) \leq \sum_{i=2}^{r+1} \lambda_{i}(L)
$$

follows from the ordering $\lambda_{2}(L) \leq \lambda_{3}(L) \leq \cdots \leq \lambda_{n}(L)$, and therefore optimal graphs must obey

$$
s_{\lambda}\left(W_{r}\right) \leq \frac{W_{r}}{r}=\frac{1}{r} \sum_{i=2}^{r+1} \lambda_{i}(L) .
$$

The bound is achievable with equality for complete graphs, which have $r \lambda_{2}(L)=W_{r}=n r$, as per Lem. 1 that follows.

Lemma 1: The complete graph on $n$ vertices, $n>2$, has the following Laplacian eigenspectrum: $\lambda_{1}=0, \lambda_{2}=\cdots=$ $\lambda_{n}=n$.

Proof: The complete graph has adjacency matrix $A$ that is all-ones except the diagonal which is all-zeros. Hence the graph Laplacian $L$ has -1 in all positions except the diagonal and has the value $n-1$ on the diagonal. Computing the eigenspectrum gives the desired result.

\section{Computational Complexity and Relaxation}

In this section, we prove that finding optimal networks is computationally hard, cf. [47], and propose a natural relaxation that is a reverse convex program.

\section{A. Proving that Network Design is Hard}

Probs. 1 and 2 are optimizations over graph Laplacians, which are discrete objects. A Laplacian matrix is symmetric, positive semidefinite, each row sums to zero, and its offdiagonal elements are either zero or minus one. Conversely, if $L$ is any $n \times n$ matrix that satisfies these conditions, then it is the Laplacian of some graph on $n$ nodes [48]. The set of Laplacian matrices

$$
\left\{L \in \mathbb{R}^{n \times n}: L=L^{T}, L \succeq 0, L \overrightarrow{1}=0, L_{i j} \in\{0,-1\}, i \neq j\right\}
$$


is denoted by $\mathcal{L}$. Since $\mathcal{L}$ is a discrete space, the optimization problems are integer programs.

The algebraic connectivity with wiring economy problem is now characterized using computational complexity theory [49], but this requires casting the optimization problem as a decision problem.

Before proceeding, define a decision version of the optimal algebraic connectivity optimization problem without any wiring cost constraints. The transformation of an optimization problem into a decision problem is done by imposing a bound on the value to be optimized.

Problem 3: Decide Algebraic Connectivity

- Given an undirected graph $G=(V, E)$, a non-negative integer $k$, and a non-negative threshold $\theta$, and letting $E^{c}$ be the complement of the edge set,

- Seek a subset $B \subseteq E^{c}$ of size $|B| \leq k$ such that the graph $H=(V, E \cup B)$ satisfies $\lambda_{2}(H) \geq \theta$.

Now we impose wiring costs on this decision problem to obtain the problem of interest.

Problem 4: DECIDE AlgEBRAIC CONNECTIVITY WITH WIRING ECONOMY

- Given an undirected graph $G=(V, E)$, a non-negative integer $k$, a non-negative threshold $\theta$, and a non-negative wiring cost $W_{r}$,

- Seek a subset $B \subseteq E^{c}$ of size $|B| \leq k$ such that the graph $H=(V, E \cup B)$ satisfies $\lambda_{2}(H) \geq \theta$ and $\sum_{i=2}^{r+1} \lambda_{i}(H) \leq$ $W_{r}$.

The first thing that can be established is that when given a solution to Prob. 4, it may be verified in polynomial time.

Theorem 3: Problem 4 is in class NP.

Proof: Eigenvalues of a matrix of size $n \times n$ can be computed in polynomial time $O\left(n^{3}\right)$. This need be done only once to verify the algebraic connectivity and wiring cost requirements and so the result follows.

Although a solution to Prob. 4 can be verified in polynomial time, finding a solution may be difficult. We prove the computational complexity of finding a solution.

Theorem 4: Problem 4 is NP-complete.

Proof: Prob. 4 is in class NP, by Thm. 3 .

Moreover, we know that Prob. 3 is NP-complete, by the main result of [50]. So we would like to use a reduction from Prob. 4 to Prob. 3 to prove the desired result.

Restrict Prob. 4 to Prob. 3 by only allowing instances having $W_{r}=n r$. This is the largest wiring cost possible for a graph with $n$ vertices (achieved by a complete graph, Lem. 1). Since Prob. 3 is a restriction of Prob. 4 that can be accomplished in polynomial time, the result is a consequence of the restriction reduction [49].

\section{B. Natural Relaxation is a Reverse Convex Program}

Given that optimal network design is NP-hard, we now develop a computationally efficient relaxation.

Let $\mathcal{C} \mathcal{L}$ be the convex hull of the set of all Laplacian matrices, $\mathcal{L}$. $\mathcal{C} \mathcal{L}$ is the set of symmetric, positive semidefinite matrices, with zero row sums, and off-diagonal elements between minus one and zero:

$\left\{L \in \mathbb{R}^{n \times n}: L=L^{T}, L \succeq 0, L \overrightarrow{1}=0,-1 \leq L_{i j} \leq 0\right.$ for $\left.i \neq j\right\}$
The natural relaxation is to consider Prob. 1, but with this larger feasible set.

The main result of this section is to show that the natural relaxation is a reverse convex program, defined as follows.

Definition 1 ( [51]): The optimization problem

$$
\begin{gathered}
\min f(x) \\
\text { subject to } x \in D \backslash C
\end{gathered}
$$

is a reverse convex program when $f(x)$ is a convex function, $D$ is a closed convex set, $C$ is an open convex set, and $D$ and $C$ are given by explicit convex inequalities.

Before proving the main result, we prove some auxiliary convexity results. Invoking a variational form of the CourantFischer theorem, $\lambda_{2}$ is a concave function.

Proposition 1: The algebraic connectivity $\lambda_{2}$ is a concave function of $L$ on $\mathcal{C} \mathcal{L}$.

Proof: Each $L \in \mathcal{C} \mathcal{L}$ is positive semidefinite and has $\lambda_{1}(L)=0$, with corresponding eigenvector $\overrightarrow{1}$. Thus $\lambda_{2}(L)$ may be expressed as

$$
\lambda_{2}(L)=\inf \left\{\vec{x}^{T} L \vec{x}:\|\vec{x}\|_{2}=1 \text { and } \overrightarrow{1}^{T} \vec{x}=0\right\} .
$$

For each $\vec{x} \in \mathbb{R}^{n}$ that satisfies $\|\vec{x}\|_{2}=1$ and $\overrightarrow{1}^{T} \vec{x}=0, \vec{x}^{T} L \vec{x}$ is a linear (and therefore also concave) function of $L$. The formula shows that $\lambda_{2}$ is the infimum of a family of concave functions in $L$, and is therefore also a concave function of $L$.

A generalization of Prop. 1 is also true and is proven in the same manner.

Proposition 2: The sum of the $k$ smallest eigenvalues of $L$,

$$
g(L)=\sum_{i=2}^{k} \lambda_{i}(L)
$$

is a concave function of $L$.

Proof: Since $\lambda_{1}(L)=0$, the sum of the smallest eigenvalues may be expressed in variational form as

$$
\begin{aligned}
g(L) & =\sum_{i=2}^{k} \lambda_{i}(L)=\sum_{i=1}^{k} \lambda_{i}(L) \\
& =\inf \left\{\text { trace }\left[S^{T} L S\right]: S \in \mathbb{R}^{n \times k} \text { and } S^{T} S=I\right\} .
\end{aligned}
$$

Note that $S^{T} L S$ is a linear (and therefore also concave) function of $L$. The formula shows that $g(L)$ is the infimum of a family of concave functions in $L$, and is therefore also a concave function of $L$.

Recall Prob. 1, but now consider matrices in $\mathcal{C} \mathcal{L}$ rather than in $\mathcal{L}$.

Theorem 5: Considering $L \in \mathcal{C} \mathcal{L}$, the optimization problem:

$$
\begin{gathered}
\max \lambda_{2}(L) \\
\text { subject to } \sum_{i=2}^{r+1} \lambda_{i}(L) \leq W_{r}
\end{gathered}
$$

is a reverse convex program.

Proof: Prop. 1 yields the convexity of the objective function. Prop. 2 implies that the feasible set is the difference between a closed convex set and an open convex set given by explicitly convex inequalities. Together, they prove the desired result. 


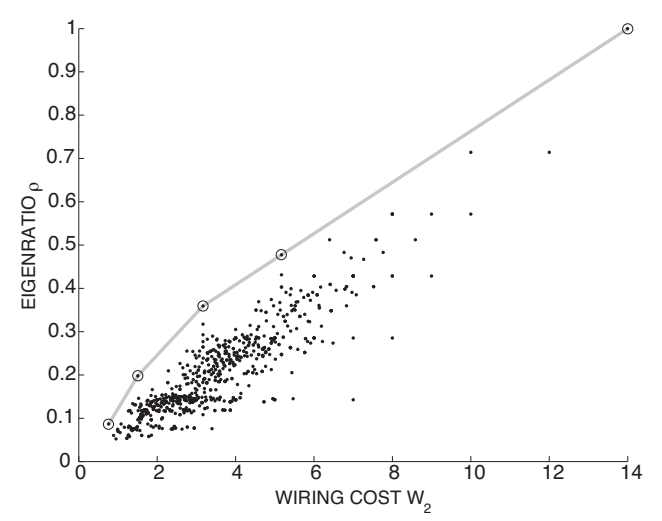

Fig. 1. Eigenratio as a function of quadratic wiring cost under optimal non-trivial placement in $\mathbb{R}^{2}$ for all connected graphs on $n=7$ vertices. The upper convex hull of these achievable functionality-cost points is denoted by the gray line. Points on the upper convex hull are circled.

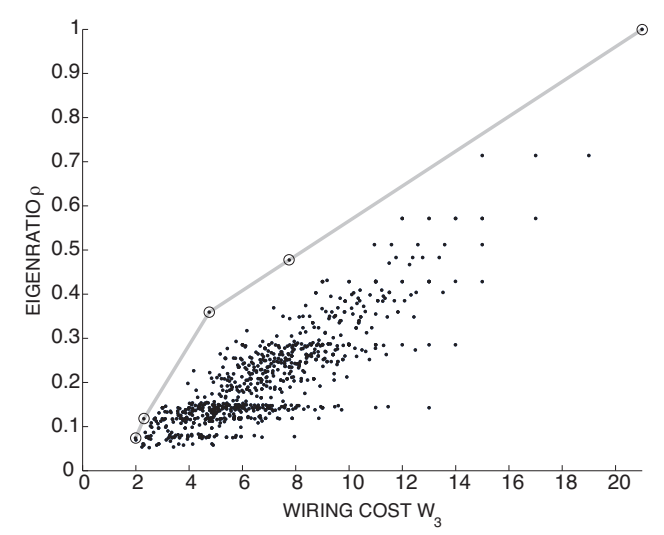

Fig. 2. Eigenratio as a function of quadratic wiring cost under optimal non-trivial placement in $\mathbb{R}^{3}$ for all connected graphs on $n=7$ vertices. The upper convex hull of these achievable functionality-cost points is denoted by the gray line. Points on the upper convex hull are circled.

Without the wiring cost constraint, the natural relaxation would have been a convex program [43]. There are several standard techniques for solving reverse convex programs [51][53], which may be used for the relaxed problem. A suitable heuristic like rounding may be used to convert a solution for the relaxed problem into a solution for Prob. 1.

\section{Optimal Networks}

Despite NP-completeness, optimal networks of small size can be designed by exhaustive enumeration. We carry out this design procedure and find exactly optimal networks, so as to gain insight into the nature of optimal designs. We could list all connected unlabeled graphs on $n$ nodes [54, A001349] and calculate their cost and functionality values [55], but since graphs can be cospectral, the number of graphs that need be checked is a much smaller number [56, Tab. 1].

Besides the sense of optimality defined in Probs. 1 and 2, a stronger sense of optimality - seeking points on the upper convex hull of functionality-cost pairs - is considered.

First, optimal networks of size $n=7$ are computed. Fig. 1 shows the eigenratio as a function of optimized 2-dimensional wiring cost for all connected graphs. The upper convex hull of achievable functionality-cost points, the boundary of optimal-

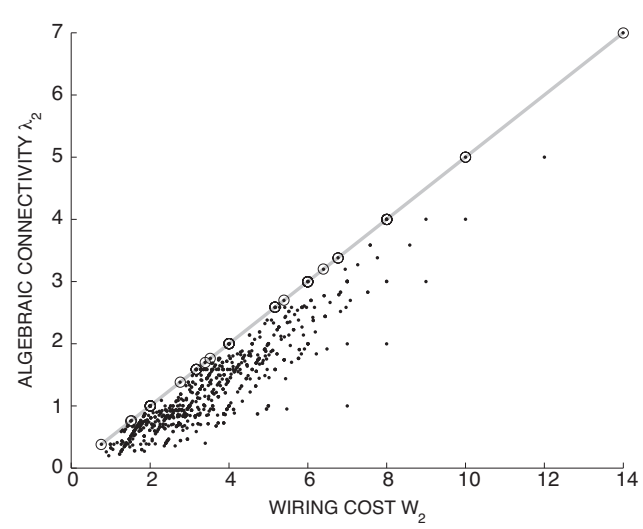

Fig. 3. Algebraic connectivity as a function of quadratic wiring cost under optimal non-trivial placement in $\mathbb{R}^{2}$ for all connected graphs on $n=7$ vertices. The upper convex hull of these achievable functionality-cost points is denoted by the gray line. Points on the upper convex hull are circled.

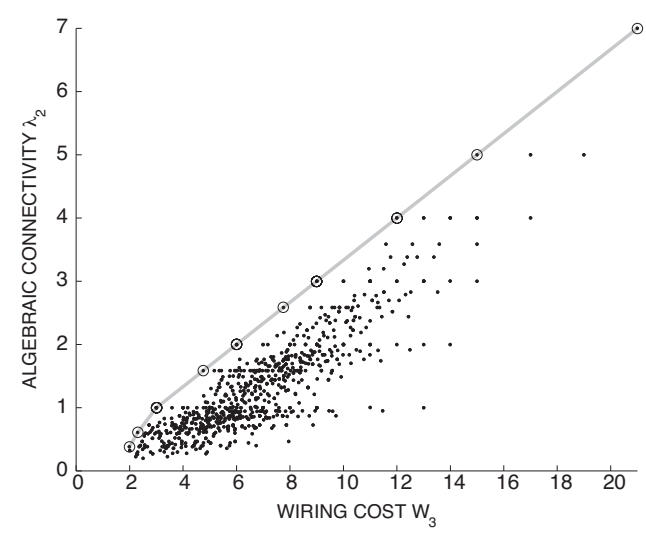

Fig. 4. Algebraic connectivity as a function of quadratic wiring cost under optimal non-trivial placement in $\mathbb{R}^{3}$ for all connected graphs on $n=7$ vertices. The upper convex hull of these achievable functionality-cost points is denoted by the gray line. Points on the upper convex hull are circled.

ity, is also shown. Fig. 2 shows the eigenratio as a function of optimized 3-dimensional wiring cost.

Considering algebraic connectivity, Fig. 3 shows $\lambda_{2}(L)$ as a function of optimized 2-dimensional wiring cost $W_{2}$; the upper convex hull is the straight line $r \lambda_{2}(L)=W_{r}$, in this case $2 \lambda_{2}=W_{2}$, which is also the upper bound from Thm. 2. Fig. 4 shows $\lambda_{2}(L)$ as a function of optimized 3-dimensional wiring cost $W_{3}$. Again, the straight line upper bound is evident.

The five optimal networks in $\mathbb{R}^{2}$, by upper convex hull for eigenratio, are shown in Fig. 6; they are drawn in a minimum wiring cost configuration in order of increasing wiring cost. Three of these five graphs are also optimal in $\mathbb{R}^{3}$.

Since insight from neuronal network design was used in developing design principles, we note the similarity between optimal designed networks and typical neuronal circuits; Fig. 5 shows the primary motor control circuit for the worm $C$. elegans with experimentally measured connectivity and neuron position [16].

The optimal networks for the eigenratio problem in $\mathbb{R}^{2}$ are a strict subset of the optimal networks for the algebraic connectivity problem in $\mathbb{R}^{2}$. There are 62 non-isomorphic network graphs that lie on the upper convex hull; some are shown in Figs. 6 and 7. That optimal networks for Prob. 2 are 
TABLE I

NUMBER OF OPTIMAL NETWORKS IN $\mathbb{R}^{2}$

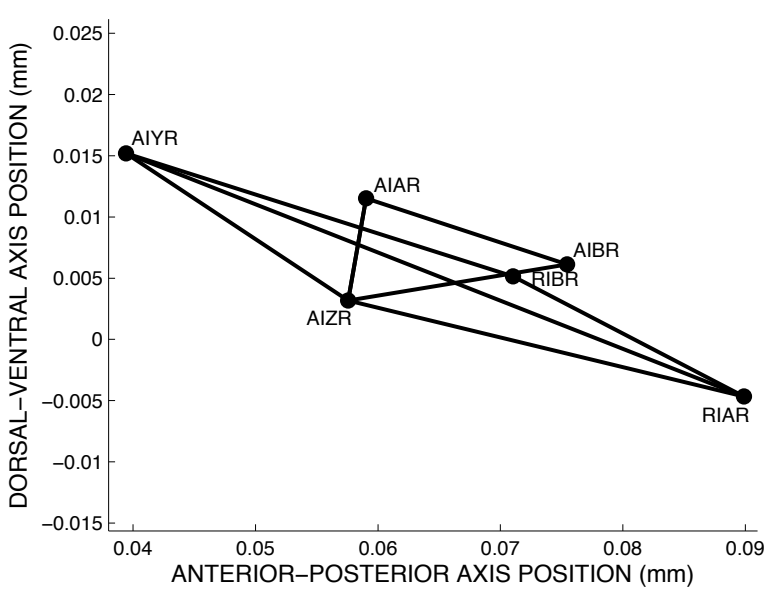

Fig. 5. A C. elegans subcircuit used for navigation control.

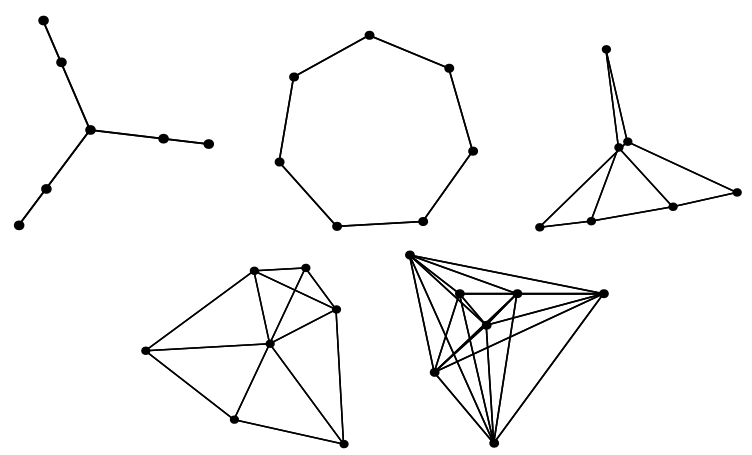

Fig. 6. All networks that are on the upper convex hull of achievable eigenratio-cost points in $\mathbb{R}^{2}$. Networks drawn in a minimal wiring cost configuration (note that this may not be unique, even up to translation/rotation if some eigenvalues have multiplicity greater than 1 ).
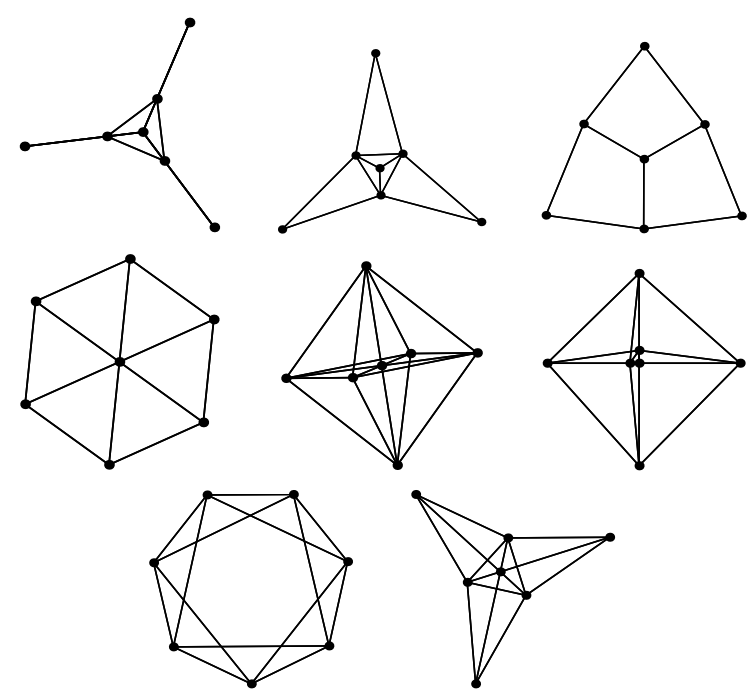

Fig. 7. Some other networks that are on the upper convex hull of achievable algebraic connectivity-cost points in $\mathbb{R}^{2}$. Networks drawn in a minimal wiring cost configuration (note that this may not be unique, even up to translation/rotation if some eigenvalues have multiplicity greater than 1).

\begin{tabular}{rrrrr}
\hline$n$ & $k(n)$ & $K_{\lambda}(n)$ & $K_{\rho}(n)$ & $K_{\lambda \cap \rho}(n)$ \\
\hline 2 & 1 & 1 & 1 & 1 \\
3 & 2 & 2 & 2 & 2 \\
4 & 6 & 3 & 3 & 3 \\
5 & 21 & 8 & 3 & 3 \\
6 & 112 & 22 & 4 & 4 \\
7 & 853 & 62 & 5 & 5 \\
8 & 11117 & 231 & 6 & 6 \\
\hline
\end{tabular}

TABLE II

NUMBER OF OPTIMAL NETWORKS IN $\mathbb{R}^{3}$

\begin{tabular}{rrrrr}
\hline$n$ & $k(n)$ & $K_{\lambda}(n)$ & $K_{\rho}(n)$ & $K_{\lambda \cap \rho}(n)$ \\
\hline 2 & 1 & 1 & 1 & 1 \\
3 & 2 & 2 & 2 & 2 \\
4 & 6 & 3 & 3 & 3 \\
5 & 21 & 2 & 2 & 2 \\
6 & 112 & 10 & 9 & 9 \\
7 & 853 & 21 & 5 & 5 \\
8 & 11117 & 61 & 6 & 4 \\
\hline
\end{tabular}

a strict subset of optimal networks for Prob. 1 for $n=7$ is an example of assertions that optimizing $\lambda_{2}$ leads to optimal $\rho$ [21], [35].

The solution containment property holds for small $n$ but does not hold in general. In Tabs. I and II we compute the number of connected graphs $k(n)$ [54, A001349], how many are optimal for algebraic connectivity $K_{\lambda}(n)$, how many are optimal for eigenratio $K_{\rho}(n)$, and how many optimal graphs for eigenratio are also optimal for algebraic connectivity $K_{\lambda \cap \rho}(n)$. The containment property holds for all but one row in the tables. It does not hold for networks in $\mathbb{R}^{3}$ of size $n=8$, since $K_{\lambda \cap \rho}(8)<K_{\rho}(n)$.

The counterexample to the containment property is displayed in Figs. 8 and 9 with red squares. These figures demonstrate networks optimal for the eigenratio-cost tradeoff are good for the algebraic connectivity-cost tradeoff.

\section{RANDOM NETWORKS}

As an alternative to explicit network structure design, we show graphs chosen at random perform well. Olfati-Saber previously studied the algebraic connectivity of graphs created by randomly rewiring regular lattices [57], along the lines of the Watts-Strogatz small-world graph ensemble. The ErdösRényi random graph ensemble is obtained when all edges are randomly rewired, so that any edge is an i.i.d. Bernoulli random variable.

\section{A. Simulations}

We ran simulations with Erdös-Rényi random graphs and measured tradeoffs between convergence speed and wiring cost. Figures 10 and 11 display the algebraic connectivities and wiring costs of 100 random networks each of size $n=500$ for several different edge existence probabilities $\kappa$. The upper bound from Thm. 2 is shown for comparison. As we can see, random networks perform well. Figs. 12 and 13 display the eigenratios and wiring costs of these random networks. 


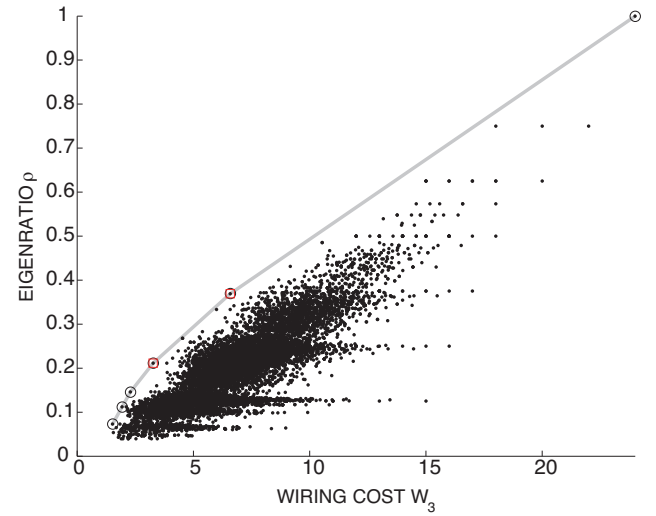

Fig. 8. Eigenratio as a function of quadratic wiring cost under optimal non-trivial placement in $\mathbb{R}^{3}$ for all connected graphs on $n=8$ vertices. The upper convex hull of these achievable functionality-cost points is denoted by the gray line. Points on the upper convex hull are circled; two of these points are further marked by red squares.

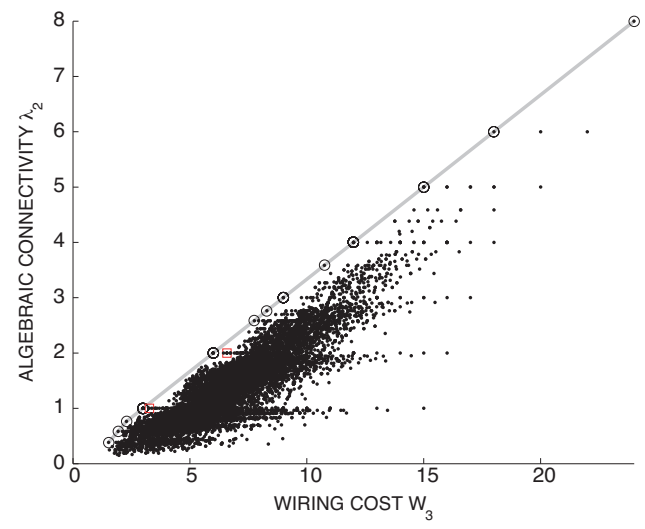

Fig. 9. Algebraic connectivity as a function of quadratic wiring cost under optimal non-trivial placement in $\mathbb{R}^{3}$ for all connected graphs on $n=8$ vertices. The upper convex hull of these achievable functionality-cost points is denoted by the gray line. Points on the upper convex hull are circled. Red squares give the performance of the networks marked in Figure 8.

\section{B. Random Matrix Theoretic Characterization}

Beyond simulation, we use random matrix theory [58], [59] to prove the following concentration results.

Theorem 6 ([60], [61]): The algebraic connectivity $\lambda_{2}(L)$ and Laplacian spectral radius $\lambda_{n}(L)$ of an Erdös-Rényi random graph with $n$ vertices and probability $\kappa$ of an edge being present asymptotically almost surely satisfy the following. For any $\varepsilon>0$,

$$
\kappa n-f_{\varepsilon}^{+}(n)<\lambda_{2}(L)<\kappa n-f_{\varepsilon}^{-}(n)
$$

and

$$
\kappa n+f_{\varepsilon}^{-}(n)<\lambda_{n}(L)<\kappa n+f_{\varepsilon}^{+}(n),
$$

where

$$
f_{\varepsilon}^{+}(n)=\sqrt{(2+\varepsilon) \kappa(1-\kappa) n \log n}
$$

and

$$
f_{\varepsilon}^{-}(n)=\sqrt{(2-\varepsilon) \kappa(1-\kappa) n \log n} .
$$

We use this result to prove the following theorem.

Theorem 7: The Laplacian eigenvalues $\lambda_{i}(L)$ of an ErdösRényi random graph with $n$ vertices and probability $\kappa$ of an

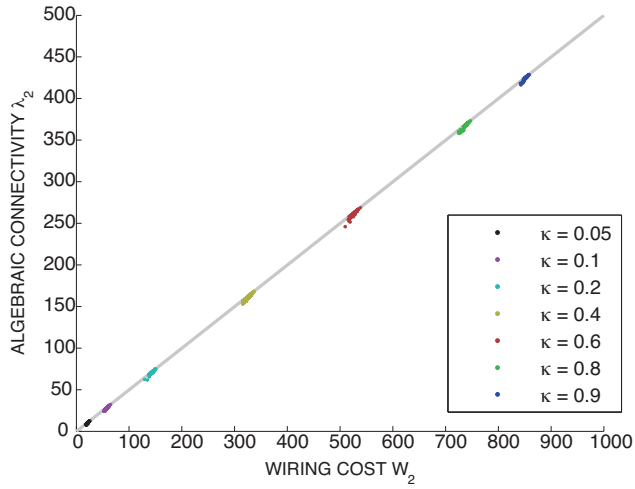

Fig. 10. Algebraic connectivity as a function of quadratic wiring cost under optimal non-trivial placement in $\mathbb{R}^{2}$ for 100 random graphs each on $n=500$ vertices for several different values of $\kappa$. An upper bound (that is also an approximation) is denoted by the gray line.

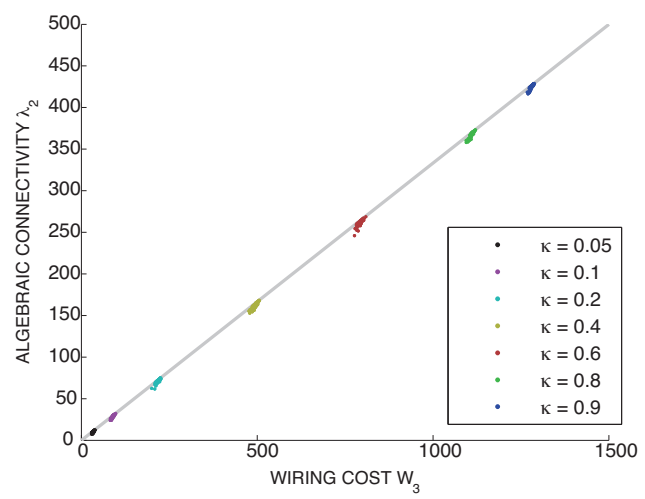

Fig. 11. Algebraic connectivity as a function of quadratic wiring cost under optimal non-trivial placement in $\mathbb{R}^{3}$ for 100 random graphs each on $n=500$ vertices for several different values of $\kappa$. An upper bound (that is also an approximation) is denoted by the gray line.

edge being present asymptotically almost surely satisfy the following. For any $\varepsilon>0$,

$\kappa n-f_{\varepsilon}^{+}(n)<\lambda_{2}(L) \leq \lambda_{3}(L) \leq \cdots \leq \lambda_{n}(L)<\kappa n+f_{\varepsilon}^{+}(n)$.

Proof: Follows directly from Thm. 6 by noting that $f_{\varepsilon}^{-}(n)<f_{\varepsilon}^{+}(n)$ and the ordering $\lambda_{2}(L) \leq \cdots \leq \lambda_{n}(L)$.

These concentration results imply that a large network constructed according to the Erdös-Rényi ensemble will almost surely behave like any other. Moreover, the convergence speed parameters will be approximately given by relatively simple functions of $n$ and $\kappa$ :

$$
\lambda_{2}(L) \approx \kappa n-\sqrt{2 \kappa(1-\kappa) n \log n}
$$

and

$$
\rho(L) \approx \frac{\kappa n-\sqrt{2 \kappa(1-\kappa) n \log n}}{\kappa n+\sqrt{2 \kappa(1-\kappa) n \log n}} .
$$

In fact, the a.a.s. differences $\lambda_{2}(L)-\lambda_{3}(L)$ and $\lambda_{2}(L)-$ $\lambda_{4}(L)$ are small, cf. [60], [62], and therefore approximation (11) can be used for $\lambda_{3}(L)$ and $\lambda_{4}(L)$. Approximate wiring costs are computed using (11) and plotted in Figs. 10-13.

The upper bound and approximation coincide for algebraic connectivity, so random networks are provably good. 


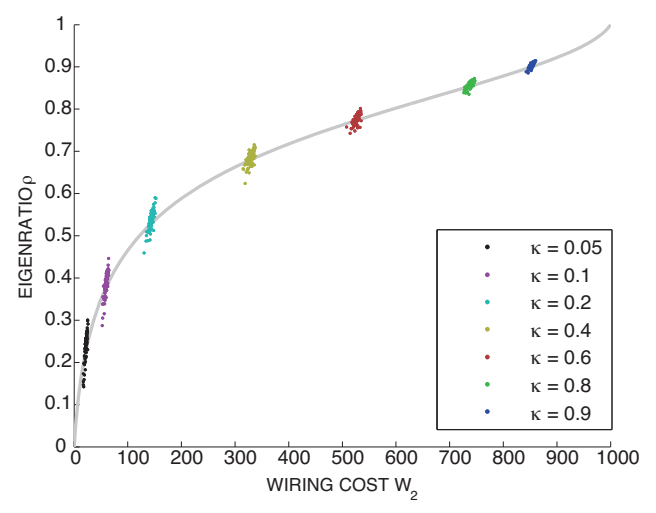

Fig. 12. Eigenratio as a function of quadratic wiring cost under optimal nontrivial placement in $\mathbb{R}^{2}$ for 100 random graphs each on $n=500$ vertices for several different values of $\kappa$. An approximation is denoted by the gray line.

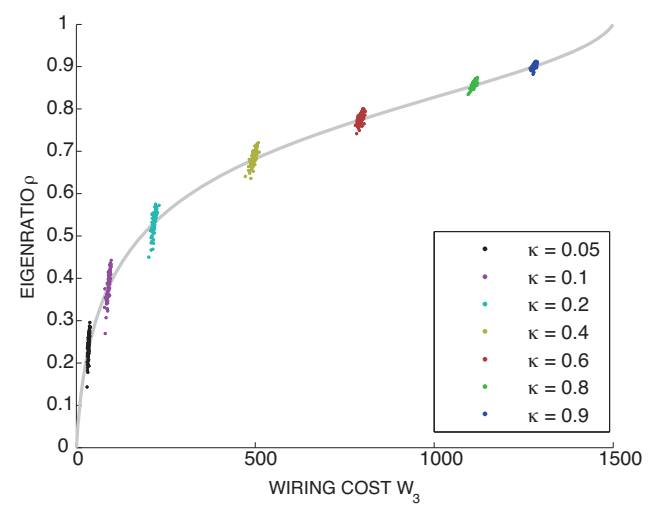

Fig. 13. Eigenratio as a function of quadratic wiring cost under optimal nontrivial placement in $\mathbb{R}^{3}$ for 100 random graphs each on $n=500$ vertices for several different values of $\kappa$. An approximation is denoted by the gray line.

\section{DISCUSSION}

Designing inference networks with physical communication infrastructures is a key engineering problem. Neuronal networks have faced evolutionary pressure to have efficient physical organization and so they provide design insights for the engineering problem. Following a mathematically precise form of the neuroscientific wiring economy principle and a formalization of network functionality as convergence speed for a broad class of message-passing algorithms, we established optimization methods to determine optimal networks.

Using an enumerative strategy for the NP-complete problem, some optimal networks were found. A reverse convex program relaxation was developed as a principled method for designing larger networks that are close to optimal, though precise characterization remains to be done. Random design of networks using the Erdös-Rényi graph ensemble also found good performance. It would be of further interest to test other random graph ensembles and to compare optimal networks to networks that occur in nature beyond the brain.

More generally, we demonstrated that concepts and insights from biological networks such as the wiring economy principle can be fruitfully applied to communication network design.

\section{ACKNOWLEDGMENT}

Discussions with J. M. F. Moura, D. B. Chklovskii, S. K. Mitter, S. Kar, J. Xavier, A. Olshevsky, P. Parrilo, and V. K. Goyal are appreciated.

\section{REFERENCES}

[1] E. Bullmore and O. Sporns, "The economy of brain network organization," Nat. Rev. Neurosci., vol. 13, no. 5, pp. 336-349, May 2012.

[2] S. Ramón y Cajal, La Textura del Sistema Nerviosa del Hombre y los Vertebrados. Springer, 1899.

[3] A. Cowey, "Cortical maps and visual perception," Quart. J. Exp. Psychol., vol. 31, no. 1, pp. 1-17, 1979.

[4] D. B. Chklovskii and A. A. Koulakov, "Maps in the brain: What can we learn from them?" Annu. Rev. Neurosci., vol. 27, pp. 369-392, Jul. 2004.

[5] G. Mitchison, "Neuronal branching patterns and the economy of cortical wiring," Proc.-R. Soc. Lond., Biol. Sci., vol. 245, no. 1313, pp. 151-158, Aug. 1991.

[6] C. Cherniak, M. Changizi, and D. W. Kang, "Large-scale optimization of neuron arbors," Phys. Rev. E, vol. 59, no. 5, pp. 6001-6009, May 1999.

[7] C. Cherniak, "Component placement optimization in the brain," $J$. Neurosci., vol. 14, no. 4, pp. 2418-2427, Apr. 1994.

[8] D. C. Van Essen, "A tension-based theory of morphogenesis and compact wiring in the central nervous system," Nature, vol. 385, no. 6614, pp. 313-318, Jan. 1997.

[9] D. B. Chklovskii, T. Schikorski, and C. F. Stevens, "Wiring optimization in cortical circuits," Neuron, vol. 34, no. 3, pp. 341-347, Apr. 2002.

[10] V. A. Klyachko and C. F. Stevens, "Connectivity optimization and the positioning of cortical areas," Proc. Natl. Acad. Sci. U.S.A., vol. 100, no. 13 , pp. 7937-7941, Jun. 2003.

[11] D. B. Chklovskii, "Exact solution for the optimal neuronal layout problem," Neural Comput., vol. 16, no. 10, pp. 2067-2078, Oct. 2004.

[12] B. L. Chen, D. H. Hall, and D. B. Chklovskii, "Wiring optimization can relate neuronal structure and function," Proc. Natl. Acad. Sci. U.S.A., vol. 103, no. 12, pp. 4723-4728, Mar. 2006.

[13] E. Z. Macosko, N. Pokala, E. H. Feinberg, S. H. Chalasani, R. A. Butcher, J. Clardy, and C. I. Bargmann, "A hub-and-spoke circuit drives pheromone attraction and social behaviour in C. elegans," Nature, vol. 458, no. 7242, pp. 1171-1176, Apr. 2009.

[14] L. R. Varshney, B. L. Chen, E. Paniagua, D. H. Hall, and D. B. Chklovskii, "Structural properties of the Caenorhabditis elegans neuronal network," PLoS Comput. Biol., vol. 7, no. 2, p. e1001066, Feb. 2011.

[15] D. George and J. Hawkins, "Belief propagation and wiring length optimization as organizing principles for cortical microcircuits," Redwood Neuroscience Institute, Tech. Rep., 2005.

[16] M. Kaiser and C. C. Hilgetag, "Nonoptimal component placement, but short processing paths, due to long-distance projections in neural systems," PLoS Comput. Biol., vol. 2, no. 7, p. e95, Jul. 2006.

[17] A. Raj and Y.-H. Chen, "The wiring economy principle: Connectivity determines anatomy in the human brain," PLOS ONE, vol. 6, no. 9, p. e14832, 2011

[18] M. T. Gastner and M. E. J. Newman, "The spatial structure of networks," Eur. Phys. J. B, vol. 49, no. 2, pp. 247-252, Jan. 2006.

[19] _ - "Shape and efficiency in spatial distribution networks," J. Stat. Mech., vol. 2006, no. 1, pp. 247-252, Jan. 2006.

[20] R. Parsani and R. Gandhi, "Building sensor networks using powerline communication," EE Times, Apr. 2010.

[21] A. A. Rad, M. Jalili, and M. Hasler, "Efficient rewirings for enhancing synchronizability of dynamical networks," Chaos, vol. 18, no. 3, p. 037104, Sep. 2008.

[22] J. A. Davis, R. Venkatesan, A. Kaloyeros, M. Beylansky, S. J. Souri, K. Banerjee, K. C. Saraswat, A. Rahman, R. Reif, and J. D. Meindl, "Interconnect limits on gigascale integration (GSI) in the 21st century," Proc. IEEE, vol. 89, no. 3, pp. 305-324, Mar. 2001.

[23] R. Ho, K. W. Mai, and M. A. Horowitz, "The future of wires," Proc. IEEE, vol. 89, no. 4, pp. 490-504, Apr. 2001.

[24] M. A. Sivilotti, "Wiring considerations in analog VLSI systems, with application to field-programmable networks," Ph.D. thesis, California Institute of Technology, Pasadena, 1991.

[25] J. A. Davis, V. K. De, and J. D. Meindl, "A stochastic wire-length distribution for gigascale integration (GSI)-Part I: Derivation and validation," IEEE Trans. Electron Devices, vol. 45, no. 3, pp. 580-589, Mar. 1998. 
[26] — "A stochastic wire-length distribution for gigascale integration (GSI) - Part II: Applications to clock frequency, power dissipation, and chip size estimation," IEEE Trans. Electron Devices, vol. 45, no. 3, pp. 590-597, Mar. 1998.

[27] D. Stroobandt, A Priori Wire Length Estimates for Digital Design. Boston: Kluwer Academic Publishers, 2001.

[28] T. N. Theis, "The future of interconnection technology," IBM J. Res. Develop., vol. 44, no. 3, pp. 379-390, May 2000.

[29] J. D. Meindl, J. A. Davis, P. Zarkesh-Ha, C. S. Patel, K. P. Martin, and P. A. Kohl, "Interconnect opportunities for gigascale integration," IBM J. Res. Develop., vol. 46, no. 2/3, pp. 245-263, Mar.-May 2002.

[30] J. Diaz, J. Petit, and M. Serna, "A survey of graph layout problems," ACM Comput. Surv., vol. 34, no. 3, pp. 313-356, Sep. 2002.

[31] P. Frankl and H. Maehara, "The Johnson-Lindenstrauss lemma and the sphericity of some graphs," J. Comb. Theory, Ser. A, vol. 44, no. 3, pp. 355-362, Jun. 1987.

[32] L. Lovász, "On the Shannon capacity of a graph," IEEE Trans. Inf. Theory, vol. IT-25, no. 1, pp. 1-7, Jan. 1979.

[33] R. Olfati-Saber, J. A. Fax, and R. M. Murray, "Consensus and cooperation in networked multi-agent systems," Proc. IEEE, vol. 95, no. 1, pp. 215-233, Jan. 2007.

[34] P. Denantes, F. Bénézit, P. Thiran, and M. Vetterli, "Which distributed averaging algorithm should I choose for my sensor network?" in Proc. 27th IEEE Conf. Computer Commun. (INFOCOM 2008), Apr. 2008, pp. 986-994.

[35] S. Kar, S. Aldosari, and J. M. F. Moura, "Topology for distributed inference on graphs," IEEE Trans. Signal Process., vol. 56, no. 6, pp. 2609-2613, Jun. 2008.

[36] L. Xiao and S. Boyd, "Fast linear iterations for distributed averaging," Syst. Control Lett., vol. 53, no. 1, pp. 65-78, Sep. 2004.

[37] M. Barahona and L. M. Pecora, "Synchronization in small-world systems," Phys. Rev. Lett., vol. 89, no. 5, p. 054101, Jul. 2002.

[38] L. Donetti, P. I. Hurtado, and M. A. Muñoz, "Entangled networks, synchronization, and optimal network topology," Phys. Rev. Lett., vol. 95, no. 18 , p. 188701 , Oct. 2005

[39] K. M. Hall, "An $r$-dimensional quadratic placement algorithm," Manage. Sci., vol. 17, no. 3, pp. 219-229, Nov. 1970.

[40] H. J. Landau, "Sampling, data transmission, and the Nyquist rate," Proc. IEEE, vol. 55, no. 10, pp. 1701-1706, Oct. 1967.

[41] R. Olfati-Saber and R. M. Murray, "Consensus problems in networks of agents with switching topology and time-delays," IEEE Trans. Autom. Control, vol. 49, no. 9, pp. 1520-1533, Sep. 2004.

[42] A. Olshevsky and J. N. Tsitsiklis, "Convergence rates in distributed consensus and averaging," in Proc. 45th IEEE Conf. Decision Control, Dec. 2006, pp. 3387-3392.

[43] A. Ghosh and S. Boyd, "Growing well-connected graphs," in Proc. 45th IEEE Conf. Decision Control, Dec. 2006, pp. 6605-6611.

[44] A. Hagberg and D. A. Schult, "Rewiring networks for synchronization," Chaos, vol. 18, no. 3, p. 037105, Sep. 2008.

[45] M. Jalili and A. A. Rad, "Comment on "rewiring networks for synchronization"," Chaos, vol. 19, no. 2, p. 028101, May 2009.

[46] S. Belhaiza, N. M. M. de Abreu, P. Hansen, and C. S. Oliveira, "Variable neighborhood search for extremal graphs. XI. bounds on algebraic connectivity," in Graph Theory and Combinatorial Optimization, D. Avis, A. Hertz, and O. Marcotte, Eds. New York: Springer, 2005, pp. 1-16.

[47] D. S. Johnson, J. K. Lenstra, and A. H. G. Rinnooy Kan, "The complexity of the network design problem," Networks, vol. 8, no. 4, pp. 279-285, Winter 1978 .

[48] A. Ghosh and S. Boyd, "Upper bounds on algebraic connectivity via convex optimization," Linear Algebra Appl., vol. 418, no. 2-3, pp. 693707, Oct. 2006.

[49] M. R. Garey and D. S. Johnson, Computers and Intractability: A Guide to the Theory of NP-Completeness. San Francisco: W. H. Freeman and Company, 1979.
[50] D. Mosk-Aoyama, "Maximum algebraic connectivity augmentation is NP-hard," Oper. Res. Lett., vol. 36, no. 6, pp. 677-679, Nov. 2008.

[51] H. Konno, P. T. Thach, and H. Tuy, Optimization on Low Rank Nonconvex Structures. Dordrecht: Kluwer Academic Publishers, 1996.

[52] H. Tuy, Convex Analysis and Global Optimization. Dordrecht: Kluwer Academic Publishers, 1998.

[53] J.-B. Hiriart-Urruty, "Conditions for global optimality 2," J. Global Optim., vol. 13, no. 4, pp. 349-367, Dec. 1998.

[54] N. J. A. Sloane, "The on-line encyclopedia of integer sequences." [Online]. Available: http://oeis.org/

[55] L. A. Goldberg, Efficient Algorithms for Listing Combinatorial Structures. Cambridge: Cambridge University Press, 1993.

[56] W. H. Haemers and E. Spence, "Enumeration of cospectral graphs," Eur. J. Comb., vol. 25, no. 2, pp. 199-211, Feb. 2004.

[57] R. Olfati-Saber, "Ultrafast consensus in small-world networks," in Proc. Am. Contr. Conf. (ACC 2005), Jun. 2005, pp. 2371-2378.

[58] Z. Füredi and J. Kómlos, "The eigenvalues of random symmetric matrices," Combinatorica, vol. 1, no. 3, pp. 233-241, Sep. 1981.

[59] F. Chung, L. Lu, and V. Vu, "Spectra of random graphs with given expected degrees," Proc. Natl. Acad. Sci. U.S.A., vol. 100, no. 11, pp. 6313-6318, May 2003.

[60] M. Juvan and B. Mohar, "Laplace eigenvalues and bandwidth-type invariants of graphs," J. Graph Theory, vol. 17, no. 3, pp. 393-407, Jul. 1993.

[61] B. Mohar and S. Poljak, "Eigenvalues in combinatorial optimization," in Combinatorial and Graph-Theoretic Problems in Linear Algebra, R. A. Brualdi, S. Friedland, and V. Klee, Eds. Springer-Verlag, 1993, vol. 50, pp. 107-151.

[62] B. Bollobás, "Degree sequences of random graphs," Discrete Math., vol. 33 , no. 1 , pp. 1-19, 1981.

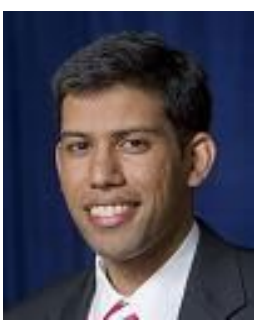

Lav R. Varshney [M] was born in Syracuse, New York on October 28, 1982. He received the B. S. degree with honors in electrical and computer engineering (magna cum laude) from Cornell University, Ithaca, New York in 2004. He received the S. M., E. $\mathrm{E}$., and $\mathrm{Ph}$. D. degrees in electrical engineering and computer science from the Massachusetts Institute of Technology (MIT), Cambridge in 2006, 2008, and 2010, respectively.

He is a research staff member at the IBM Thomas J. Watson Research Center, Yorktown Heights, NY. He was a postdoctoral associate and research assistant in the Signal Transformation and Information Representation Group in the Research Laboratory of Electronics, a National Science Foundation graduate research fellow, and a research assistant in the Laboratory for Information and Decision Systems at MIT. He was an instructor in the MIT Department of Electrical Engineering and Computer Science in Spring 2009 and a teaching assistant in Fall 2006. He was a visiting scientist at Cold Spring Harbor Laboratory, Cold Spring Harbor, New York in 2005. His research interests include neuroscience, statistical signal processing, information and coding theory, collective intelligence, and computational creativity.

Dr. Varshney is a member of Tau Beta $\mathrm{Pi}$, Eta Kappa Nu, and Sigma $\mathrm{Xi}$. He received the Jin-Au Kong Award Honorable Mention for Electrical Engineering doctoral thesis, the Ernst A. Guillemin Thesis Award for Outstanding Electrical Engineering S.M. Thesis, a best paper award at the 2012 SRII Global Conference, the Capocelli Prize at the 2006 Data Compression Conference, the Best Student Paper Award at the 2003 IEEE Radar Conference, and was a winner of the IEEE 2004 Student History Paper Contest. 